\title{
Formación en competencias digitales: un diagnóstico que da voz a los estudiantes de psicopedagogía
}

\section{Training in digital competences: a diagnosis that gives voice to psychopedagogy students}

\author{
Araceli Camacho Navarro \\ Universidad Autónoma de Querétaro \\ Araceli.camacho@uaslp.mx \\ Rolando Javier Salinas García \\ Universidad Autónoma de Querétaro \\ Javier.salinas.uaq@gmail.com
}

Recibido: $13 / 03 / 2020$

Aceptado: 03/09/2020

Publicado: 01/12/2020

\section{RESUMEN}

La práctica docente en la sociedad del conocimiento requiere de competencias digitales, esto permite que los docentes se desenvuelvan en escenarios en los que se necesita el uso reflexivo de la tecnología educativa. Es por ello que las Instituciones con énfasis en formación de especialistas en educación necesitan desarrollar las competencias digitales en sus estudiantes partiendo de un diagnóstico. El objetivo de la investigación es identificar los factores que impactan en los estudiantes al hacer uso de la tecnología por medio de las narrativas digitales, para determinar las acciones para su incorporación en el periodo formativo tratando de que sea contextualizada al alumno. Para este estudio se aplicó un cuestionario a 175 alumnos que estudian psicopedagogía en una Universidad Pública de México, complementando los resultados con el análisis de 10 narrativas autobiográficas con el instrumento denominado T-autoetnografía, que muestra la importancia que tiene la tecnología a largo de su vida haciendo una conexión con el periodo de formación; estos resultados permiten identificar las dimensiones en tecnología educativa que más impactan para lograr su incorporación y hacer uso de ella en su futura práctica profesional dando voz al estudiante y construyendo significados sobre tecnología.

\section{PALABRAS CLAVE}

Competencia digital; narrativa digital; tecnología educativa; formación inicial docente.

\section{ABSTRACT}

Teaching practices in the knowledge society require digital competences, this allows teachers to develop scenarios in which the reflexive use of educative technology is needed. That's why the Institutions with emphasis on the training of education specialists need to develop the digital skills of their students beginning from a diagnosis. The objective of this investigation is identifying the factors that impact students when making use of technology by digital narratives and thus determine the actions for their incorporation in this training period, trying to be contextualized to the student. For this study, a questionnaire was applied to 175 students and was supplemented with $10 \mathrm{~T}$-autoethnographies from a Mexican public university, these autobiographical narratives show the importance that technology has had during their lives, making a connection with the formative period. These results allow the identification of the dimensions in educative technology that are more impacting for a student in order to achieve their incorporation and use in their future professional practice giving the student a voice and building meanings about technology. 


\section{KEYWORDS}

Digital competences; digital narrative; educational technology; teacher training.

\section{CITA RECOMENDADA}

Camacho, A. y Salinas, R.J. (2020). Formación en competencias digitales: un diagnóstico que da voz a los estudiantes de Psicopedagogía. RIITE. Revista Interuniversitaria de Investigación en Tecnología Educativa, 9, 1-18. http://dx.doi.org/10.6018/riite.418331

\section{Principales aportaciones del artículo y futuras líneas de investigación:}

- El uso de la narrativa digital y la técnica denominada T-autoetnografía.

- Análisis de la conexión que existe entre la tecnología y el futuro docente desde su contexto.

- Dimensiones de análisis entre los factores que impactan al individuo para el desarrollo de las competencias digitales.

\section{INTRODUCCIÓN}

Actualmente en este mundo globalizado, ha emergido una realidad en el contexto educativo a la que no podemos ignorar y hace necesaria la transformación de los procesos de enseñanza, en las escuelas los alumnos se encuentran hiperconectados, con inmediatez al acceder a la información, y por si fuera poco este escenario, la sociedad es denominada del conocimiento, que por el simple concepto ya tiene un peso y una responsabilidad que se adjudica a la educación.

Un personaje central en la sociedad del conocimiento es el docente, por tal motivo la investigación se centra en su proceso de formación inicial, se hace un análisis de las situaciones que se están viviendo en las escuelas que se encargan de formar a los futuros docentes. Es imperativo que los docentes estén capacitados y dotados de Competencias Digitales (CD) necesarias en esta sociedad que se mueve por el desarrollo tecnológico, dichas competencias le proporcionarán herramientas para su propia práctica docente.

La práctica docente se ha visto inmersa en cambios muy importantes, uno de los factores ha sido la incorporación de las Tecnologías de la Información y de la Comunicación (TIC) en el ámbito educativo, en las Universidades encargadas de formar a formadores hay interés en la incorporación de la Tecnología Educativa (TE), ejemplo de ello es que algunas modifican sus planes de estudio agregando alguna asignatura sobre las TIC, ofertando algún curso extracurricular o materia optativa ofreciendo una forma de actualización a los alumnos.

Las Instituciones de Educación Superior con énfasis en pedagogía no están exentas de hacer cambios y actualizaciones en sus planes de estudio. Actualmente, de estas instituciones egresan profesionistas con la intención de formar parte del magisterio, por tal motivo, la integración de la TE y el desarrollo de las CD son temas que se han puesto sobre la mesa como una necesidad para su futura práctica docente. La incorporación de cualquier tecnología necesita tener conexión con quien la va a usar, para tal efecto, se plantea una técnica para dar voz a los estudiantes, favoreciendo el conocimiento y reconocimiento del uso de la TE.

De acuerdo con lo anterior, ésta investigación realizada con estudiantes de psicopedagogía de una Universidad Pública del estado de San Luis Potosí en México, tiene como objetivo hacer un diagnóstico de las $C D$ en futuros docentes identificando los factores que impactan en los estudiantes al hacer uso de la tecnología por medio de narrativas digitales. Lo anterior permitirá determinar las acciones y estrategias para el desarrollo de CD en la Formación Inicial Docente (FID) de una forma contextualizada y dando voz al alumno. Es por ello que el trabajo forma parte de la primera fase de cuatro que se desarrollan en una tesis doctoral que propone una estrategia para desarrollar las $C D$ en alumnos que se encuentran en Instituciones de Educación Superior (IES) enfocadas en FID. 
Para hacer el diagnóstico, se utiliza la narrativa digital por medio de la técnica denominada T-autoetnografía, diseñada a partir de la combinación de la tecnoautobiografía y la autoetnografía del aprendizaje para conocer de viva voz las impresiones que tienen sobre la tecnología a lo largo de su vida, conectando su proceso formativo con su vivencia a lo largo de la vida con las herramientas digitales.

En concordancia con otros trabajos, las investigaciones previas en CD en estudiantes de magisterio o en FID son relativamente escasas y se han centrado fundamentalmente en profesorado activo (Tárraga-Mínguez, Sanz-Cervera, Pastor-Cerezuela, \& Fernández-Andrés, 2017). Por lo anterior, se hace necesaria esta investigación partiendo del diagnóstico para realizar futuras investigaciones en el tema.

\subsection{Hacia la construcción de CD en los futuros docentes}

Parte de lo que se menciona en la sociedad del conocimiento es que está estrechamente relacionada con la educación que se imparte en las universidades y las prácticas que los docentes lleven a cabo en las instituciones, así mismo de ella dependerá, el desarrollo de las competencias y habilidades necesarias que prepara profesionistas altamente calificados para la producción de conocimiento científico y tecnológico, que como lo mencionan Moreno \& Velázquez (2012), y que únicamente los individuos que desarrollen dichas competencias serán incluidos en las actividades productivas, existiendo una presión económica y social para la incorporación de la tecnología en la enseñanza (Martínez, 2016, p. 19).

Actualmente se plantea un nuevo escenario para la educación, en el que adquiere una nueva dimensión: convertirse en el motor fundamental para el desarrollo económico y social. Por tradición, la educación se considera una prioridad en las políticas culturales, de bienestar social y de equidad, siendo una estrategia prioritaria en las políticas de desarrollo (Coll, 2008).

Sobre la base de las ideas anteriormente expuestas, se entiende pues, que las personas y los individuos que habitan este planeta cada día se ven más inmersos en el mundo de las TIC, por lo tanto, se hace más visible la necesidad de hacer uso de ellas y no solo eso, darles un uso correcto. El compromiso social de las universidades se hace presente ante el panorama descrito, pero también se requiere la integración de herramientas de la TE que facilite el aprendizaje en los alumnos como acompañamiento en el aula (Zúñiga, Lozano, García, \& Hernández, 2018).

En este sentido Negroponte (1995), también se manifestó mencionando que una parte significativa del aprendizaje proviene de la enseñanza, refiriéndose a esta como "una buena enseñanza con buenos profesores", entendiendo que los recursos tecnológicos pueden ayudar a simplificar la actividad de los profesores y activar a los alumnos cuando existe una integración adecuada de ella.

Las CD son señaladas por la UNESCO (2008), como una necesidad para el docente, tomando en cuenta que a los alumnos que se enfrentarán en el aula, provienen de una nueva generación, están en contacto directo con la tecnología, viven en una cultura donde la interacción y el paradigma comunicacional se basa más en la interactividad al usar un medio instantáneo y personalizable como Internet, lo que implica, una serie de cambios en el uso y comprensión de los códigos de comunicación.

No existe una "definición unánime de la Competencia Digital" (Gisbert at al., 2016, p. 76), pero se puede describir como algo que va mucho más allá de usar las herramientas tecnológicas, con ello se quiere significar que es necesario tener conocimientos y capacidades que permitan reconocer las herramientas que apoyen en el proceso de enseñanza y aprendizaje para después hacer una selección de ellas y, finalmente se puedan integrar en los programas académicos (Prendes, Gutiérrez, \& Martínez, 2018).

En la formación de los docentes, el desarrollo y fortalecimiento de las CD significa dotarlos de herramientas que les van a permitir desarrollarse profesionalmente, intercambiando información, generando redes de aprendizaje entre ellos, aunado a esto les ofrecerá facilidad en la elaboración de estrategias didácticas para crear un ambiente que favorezca el aprendizaje de sus estudiantes; sin dejar de lado que son un apoyo en la gestión y colaboración en los centros educativos a los que pertenezcan. El uso reflexivo de las TIC por parte del docente también depende de la apropiación que él haga de ellas (Valencia-Molina et al., 2016). 


\subsection{Tecnología Educativa (TE)}

La TE tiene sus orígenes dice Prendes "desde la psicología y su afán de tratar de encontrar los puentes conceptuales entre el aprendizaje y los medios de enseñanza" (2018, p. 6). En sí, dicho concepto ha ido evolucionando y tomando forma por la diversidad de áreas que se han encargado de estudiar los medios tecnológicos que se van haciendo presentes en los ambientes que se construyen en los procesos de enseñanza-aprendizaje. $Y$ es en este apartado que aprovechamos para subrayar que no son sinónimos los conceptos de TE y TIC, al hablar de TIC estamos hablando de herramientas, a diferencia de TE que es todo un corpus teórico (Serrano \& Prendes, 2016).

Sancho et al., mencionan que "no se debe considerar la TE como el último artefacto o la aplicación que mejora el procesamiento de la información” (2015, p. 18). Se recomienda tomar en cuenta otros aspectos importantes como lo son las instituciones y las relaciones que se tejen en la misma, así mismo podría extender el mito de que las tecnologías tiene el poder de mejorar la educación per se.

Conviene subrayar que la TE y su acceso todavía no es equitativa en todas las instituciones, aún en estos tiempos, podemos encontrar instituciones equipadas con una gran diversidad de estas herramientas y otras en zonas rurales en donde no se cuenta con elementos básicos como la conectividad, por ejemplo en el contexto Mexicano en el ciclo escolar 2015-2016 (Tabla 1), y la distribución del equipamiento tecnológico no es equitativo entre los distintos servicios educativos y es donde se muestra más amplia la brecha tecnológica (Tabla 2).

Tabla 1. Equipamiento tecnológico de las escuelas en México, específicamente la disponibilidad de una computadora para uso educativo y su conexión a internet

\begin{tabular}{llcc}
\hline Nivel & Cantidad & Porcentaje & $\begin{array}{c}\text { Con conexión a } \\
\text { internet }\end{array}$ \\
\hline primaria & 39,589 & $40.4 \%$ & $26,491(66.9 \%)$ \\
\hline secundaria & 27,245 & $70.1 \%$ & $18,324(67.3 \%)$ \\
\hline
\end{tabular}

Fuente: (INEE, 2017, p. 18)

Tabla 2. Disponibilidad de computadoras entre los distintos tipos de servicios de las escuelas de México

\begin{tabular}{ccc}
\hline Nivel & Tipo de servicio & $\begin{array}{c}\text { Porcentaje de al menos } \\
\text { una computadora para uso } \\
\text { educativo }\end{array}$ \\
\hline Primaria & Comunitaria & $0.04 \%$ \\
\cline { 2 - 3 } & Indígena & $23.6 \%$ \\
\hline Secundaria & General & $48.1 \%$ \\
\cline { 2 - 3 } & Comunitaria & $1.9 \%$ \\
\cline { 2 - 3 } & Para trabajadores & $32.9 \%$ \\
\cline { 2 - 3 } & Telesecundarias & $70.7 \%$ \\
\hline
\end{tabular}

Fuente: (INEE, 2017, p. 18).

De acuerdo con lo anterior, podemos darnos una idea de la realidad que impera en el contexto de México, esta realidad es a la que se enfrentarán los docentes en su práctica profesional en cuestión de TE. Algunos docentes en su aula contarán con TE que les permitirá poner en práctica la competencia digital desarrollada y algunos otros encontrarán un escenario en donde la infraestructura tecnológica es escasa o nula. Frente a esta situación, en ocasiones 
es complejo visualizar como una necesidad el desarrollo de las competencias digitales en la FID, ya sea por la misma institución, como por los estudiantes que se encuentran en ella.

\subsection{Formación Inicial Docente (FID) en TE}

Otra de las tareas prioritarias para reducir la brecha digital mencionada anteriormente son la formación adecuada del profesorado y reformar las escuelas en su pedagogía para una inclusión eficaz de la TE.

Las instituciones encargadas de la formación de los docentes no pueden cerrar los ojos a las transformaciones que han surgido en la educación, cultura, economía y hasta el ocio gracias a las TIC, el mantenerse al margen es descontextualizar la formación profesional con la práctica docente. Cada día se hace "necesaria la intervención relacionada con la TE como productos, programas, materiales, procedimientos, escenarios, procesos y otros con similitud" (De Benito \& Salinas, 2016, p. 47).

Autores como Sancho et al. (2015), indican que la formación docente sea inicial o permanente debe acercarse a las complejidades de la profesión en donde mínimo debe:

- Entender las dimensiones de su tarea en la sociedad que le ha tocado vivir.

- Desarrollar y adquirir habilidades y predisposiciones que le posibiliten el establecimiento de relaciones de aprendizaje productivas con el alumnado.

- Poder pensar alternativas a la forma tradicional de impartir la enseñanza.

- Analizar las consecuencias de los cambios producidos por las tecnologías digitales Plantearse el papel de recursos digitales en la vida y la forma de aprender y de relacionarse de sus estudiantes y su utilización en los procesos de enseñanza y aprendizaje formales.

"La labor docente enfrenta importantes desafíos y las autoridades deben acercar a los maestros los elementos para poder desempeñarse exitosamente y seguir impulsando el desarrollo de nuestro país" (SEP, 2017, p. 127).

En México existe una alta demanda de nuevos maestros, y por eso el sector educativo se vio en la necesidad de abrir sus puertas a profesionistas con formación universitaria diversa y a egresados de licenciaturas con énfasis en educación, esto tiene su grado de complejidad, por la naturaleza de las Instituciones de Educación Superior al tener autonomía en la determinación de sus planes y programas como lo señala la Constitución Política de los Estados Unidos Mexicanos (2020) en el Artículo tercero. apartado VII.

El escenario antes descrito, también dio origen a cambios en las Escuelas Normales, que hasta entonces eran por tradición las encargadas de la FID. Se llevó a cabo "La Estrategia de fortalecimiento y transformación de las Escuelas Normales" en donde los planes y programas tuvieron cambios y actualizaciones, entre estos cambios están las competencias profesionales para el uso de la innovación y la TE en su práctica docente (SEP, 2018). Lo anterior como respuesta al planteamiento pedagógico que la Secretaría de Educación había propuesto:

Para enfrentar los retos que impone el mundo globalizado del siglo XXI y hacer realidad el planteamiento educativo, es indispensable fortalecer la condición profesional de los docentes en servicio, y construir una formación inicial que garantice el buen desempeño de quienes se incorporen al magisterio. La labor docente enfrenta importantes desafíos y las autoridades deben acercar a los maestros los elementos para poder desempeñarse exitosamente y seguir impulsando el desarrollo de nuestro país (SEP, 2017, p. 127).

\subsection{Las Competencias Digitales (CD) en la FID}

Los planes de acción en donde figuran las tecnologías tienen lagunas en cuanto la integración de las TIC y desarrollo de las CD en la FID, estos temas todavía no reciben una debida atención y la información que existe sobre ellos es escasa, porque el énfasis que ha recibido se refiere a infraestructura y conectividad (Vaillant, 2013). 
Tomando en cuenta lo anterior, se entiende que la incorporación de las competencias en los planes de estudio no es una tarea fácil, esto conlleva una "revolución a nivel pedagógico que compromete un cambio metodológico a nivel académico" (Larraz at al., 2014, p. 71). Este escenario no cambia mucho cuando hablamos específicamente de las CD en la FID, en donde implica cambios desde los planes de estudio en las instituciones, hasta las guías docentes de las materias.

Vaillant (2013), distingue propuestas de cursos en FID dirigidos a estudiantes en este periodo formativo que no se encuentran explícitamente en la malla curricular o en los programas curriculares, además, estos se ofrecen también a maestros en activo, por lo tanto se constituyen como parte de la FID y formación continua. Lo anterior puede representar para los futuros docentes una desventaja ante los docentes en ejercicio.

El integrar una estrategia para el desarrollo de CD en la FID también plantea "la disyuntiva entre posicionarlas como asignaturas específicas orientadas al desenvolvimiento de habilidades básicas, o de manera transversal vinculándolas significativamente a los aspectos metodológicos y didácticos de las asignaturas, o ambas vías complementarias" (Vaillant, 2013, p. 32). Si se hace de forma transversal existe el riesgo de no encontrar a nadie que se haga responsable de la estrategia y si es a través de alguna asignatura específica podría ser vista de forma aislada al resto del programa formativo.

Las instituciones encargadas de la FID hacen esfuerzos para el desarrollo de la CD, realizando cursos, mejorando la infraestructura e incrementando el uso de dispositivos digitales. No obstante, el hecho de que la tecnología esté presente solo en áreas específicas de la FID dificulta el desarrollo de la CD y la integración de las TIC con un fin pedagógico.

De acuerdo a lo anterior, Palacios et al. (2018), sugieren las siguientes consideraciones en las universidades encargadas de formar a futuros formadores:

- La competencia digital y mediatica debe estar presente de forma interdisciplinar en el currículo.

- Un modelo eficiente para el desarrollo de las CD debe permitir a los estudiantes participar en un entorno de aprendizaje digital.

- Las nuevas tecnologías y los recursos digitales usados en la institución deben estar en constante evaluación.

- Un punto clave en la FID, es crear conciencia de la importancia de las TIC en esta etapa formativa como en la futura práctica docente en el aula.

Hablando sobre CD en la FID en América Latina, es necesario remarcar tres situaciones a las que nos enfrentamos (Brun, 2011):

1. Hay una disociación entre la información que muestran los Ministerios de Educación de algunos países con referencia a las TIC en la FID y lo que ocurre realmente en las instituciones, porque los datos que muestran, en ocasiones resultan insuficientes e incompletos.

2. En comparación con los antecedentes en formación continua para docentes en servicio, se comprueba que existe una presencia significativa menor de experiencias en integración de TIC en FID.

3. Los factores que emergen con una integración exitosa de las TIC son los que se refieren a la sostenibilidad en tiempo a las experiencias, como ejemplo la institucionalización, existencia de soporte técnico, pedagógico y financiero, transferibilidad y escalabilidad, difusión, etc.

El diagnóstico de las $\mathrm{CD}$ en las instituciones ha constituido un método para recabar información valiosa sobre el uso que se hace de la tecnología y detectar posibles necesidades, como es el caso del instrumento realizado por el grupo de investigación de la didáctica de las CCSS e Innovación pedagógica en la sociedad (INCISO), en el que fue validado y dirigido a los estudiantes de FID de infantil y primaria. El diagnóstico realizado con el instrumento diseñado por los investigadores, también tiene como finalidad conocer el uso educativo de las redes 
sociales en la formación inicial por parte de los alumnos en una Universidad no presencial y exclusivamente on line (León-Gómez, Calderón-Garrido, \& Gil-Fernandez, 2019).

\subsection{Uso de las Narrativas Digitales}

El hacer uso de narrativas en el proceso de FID, tiene como propósito reivindicar el papel del docente y su formación, se ve como pieza clave la reconstrucción de la enseñanza para la preparación de los futuros maestros, tomando en cuenta la identidad y la subjetividad en ellos, se menciona la importancia de la relación entre el contexto y consigo mismos, el maestro se cuestionará sobre sus prácticas y a su vez se preguntará por la naturaleza de la enseñanza en la comunidad (Paredes, 2014, p. 10).

Lo anterior va en en concordancia con:

Todo docente, sea cual sea su papel en el sistema educativo, precisa formación inicial y permanente. En el ámbito específico de la TE esta concepción de la formación significaría analizar con el profesorado las complejas dimensiones organizativas, simbólicas y artefactuales de las instituciones educativas actuales (Sancho et al., 2015, p. 20).

El uso de la narrativa digital trata de reivindicar la importancia de lo biográfico haciendo uso de las TIC, se inicia un diálogo entre el ayer y lo que se vive generándose un proceso activo entre ellos para después hacer una construcción social de la realidad. Además, expresa dimensiones emotivas de la experiencia, la complejidad, relaciones y singularidad de cada momento que se plasma en una narración, esto permite captar riqueza en detalles que con otras técnicas sería más complicado identificar (Bolivar \& Domingo, 2019).

El uso de la narrativa digital amplía el ámbito de estudio en TE, considerando, además del contexto instruccional, el contexto socio-cultural, familiar y personal para la contribución al entendimiento y explicación del proceso de enseñanza y aprendizaje (Solano, 2018). Así pues, Padilla-Hernández, Gámiz-Sánchez, \& Romero-López, mencionan la necesidad de encontrar estrategias para diagnosticar las necesidades formativas de la Competencia Digital Docente (CDD) reconiciendo la influencia del contexto institucional y social (2018).

Gracias al internet y a los avances tecnológicos existe una amplia gama de medios digitales, esto ha favorecido la creación de diferentes formas de llevar a cabo proyectos etnográficos, siendo estos una forma original e innovadora de realizar los diseños etnográficos sin perder las características de la etnografía tradicional (Hine, 2012).

\subsection{T-autoetnografía}

La T-autoetnografía retoma los beneficios ofrecidos por las dos técnicas narrativas (tecnoautobiografía+autoetnografía del aprendizaje) que se retoman de Paredes (2014) y Lopes et al. (2013), estas a su vez, estarán mediadas por elementos digitales (narrativa digital), entendiendo esto como un proceso etnográfico para autobservación a profundidad que ayuda a interpretar la realidad por medio de las interacciones del objeto de estudio con lo tecnológico, véase Figura 1.

$$
T \text {-autoetnografía }=\frac{\text { Tecnoautobiografía }+ \text { Autoetnografía del aprendizaje }}{\text { Narrativa digital }}
$$

Figura 1. Elementos que conforman la técnica T-autoetnografía

La tecnoautobiografía acerca el concepto de tecnología por medio de las relaciones existentes con su vida, explorando el conjunto de tecnologías que han estado alrededor del individuo y que lo expresen haciendo uso de las tecnologías digitales por medio de una narrativa multimedial descrita por Hermann (2015), como el conjunto de mensajes sonoros y visuales en los proceso educomunicativos, se dice que "la mayoría de las tecnoautobiografías realizadas por estudiantes reflejan un cambio significativo tanto en la forma de entender las dimensiones de la tecnología como de posicionarse ante ella" (Lopes et al., 2013, p. 127). 
La autoetnografía del aprendizaje reconoce la forma más propicia para desarrollar las CD partiendo de lo que identifican los sujetos al momento de llevar a cabo su propia construcción del aprendizaje, Lopes et al., la define como "el estudio de una persona llevado a cabo por ella misma" (2013, p. 128).

\section{MÉTODO}

Partiendo de nuestro posicionamiento, se utiliza el método narrativo mediante la técnica denominada $T$-autoetnografía, para obtener de viva voz de los futuros docentes las impresiones sobre la tecnología en el ambiente educativo, a su vez, identificaremos las CD que ellos consideran importantes para desarrollar en su formación inicial docente.

Con el diagnóstico hecho por medio de las T-autoetnografías vamos a obtener los beneficios de las técnicas narrativas que la conforman (tecnoautobiografía y autoetnografía del aprendizaje) generando un dialogo entre el ayer y lo que viven en el proceso de aprendizaje los alumnos en FID sobre TE y las CD, en donde se considera, el contexto instruccional, socio-cultural, familiar y personal; tratando de hacer un estudio más amplio al diagnóstico de las CD, en donde no solo se evalúe el uso de herramientas digitales, sino también obtener un panorama de influencias para el desarrollo y uso de la TE.

\subsection{Diseño}

Para este estudio se utilizó el enfoque cualitativo-interpretativo, entendiendo que trata del estudio de un todo integrado y que constituye una unidad de análisis, por su naturaleza interpretativa ayudados de la hermenéutica se trata de observar algo para darle significado. Lo anterior consiste en reconocer los elementos que impactan en los estudiantes al utilizar las TIC en el proceso de enseñanza y aprendizaje, y el apoyo que representan para su futura práctica profesional.

Se ahondó en los factores que intervienen en el desarrollo de las CD en el contexto educativo y se identifican las situaciones que ellos consideran relevantes al momento de implementar las TIC en su futura práctica docente, así mismo, se busca analizar y acercar el concepto de tecnología por medio de las relaciones existentes con su vida, que exploren el conjunto de tecnologías que han estado a su alrededor. Con este proceso reflexivo se tomarán elementos y dimensiones referentes a identidad, la forma en como se ha transformado el entorno y las implicaciones que se dan por el uso de la tecnología en la comunidad.

\subsection{Participantes}

El presente estudio se llevó a cabo en alumnos de la Licenciatura en Psicopedagogía de una Universidad Pública del Estado de San Luis Potosí, México. Se aplicó el cuestionario a 175 alumnos de un total de 241 que cursan la licenciatura en Psicopedagogía, la intención era aplicarlo a la población total de estudiantes de la licenciatura pero solamente se llevó a cabo con los que se encontraban en el aula al momento de aplicar el cuestionario, lo anterior de acuerdo a la planeación y horarios de clase. Los 175 estudiantes que contestaron pertenecen a los nueve grupos que hay en esta carrera, dos grupos por semestre de los cinco años que corresponden en la licenciatura, con excepción de los que se encuentran cursando el décimo semestre, en donde solamente es un grupo. De los jóvenes que aplicaron fueron un total de 154 mujeres y 21 hombres con un promedio de edad de 20.6 años. Específicamente los jóvenes que participaron quedaron distribuidos como sigue (Tabla 3):

Tabla 3. Distribución de los alumnos que aplicaron el cuestionario

\begin{tabular}{ccc}
\hline Semestre & $\begin{array}{c}\text { Alumnos que } \\
\text { contestaron }\end{array}$ & Total \\
\hline Segundo & 41 & 61 \\
\hline Cuarto & 50 & 60 \\
\hline
\end{tabular}




\begin{tabular}{ccc}
\hline Sexto & 43 & 59 \\
\hline Octavo & 33 & 45 \\
\hline Decimo & 8 & 16 \\
\hline Total & 175 & 241 \\
\hline
\end{tabular}

\subsection{Instrumentos}

Se utilizaron 10 (diez) T-autoetnografías para este estudio, pertenecientes a 10 alumnos de la Licenciatura en Psicopedagogía dos alumnos por cada grupo que fueron seleccionados aleatoriamente en el aula.

Las T-autoetnografías se complementaron con un cuestionario para hacer el diagnóstico de la Competencia Digital (CD) en la FID utilizado para medir las CD en la Universidad Internacional de la Rioja (UNIR), perteneciente al grupo de investigación de la didáctica de las CCSS e Innovación pedagógica en la sociedad (INCISO). Dicho cuestionario fue diseñado y validado tomando en cuenta las características de los estudiantes en grado de licenciatura y maestría (León-Gómez et al., 2019).

\subsection{Procedimiento y análisis de los datos}

Se hizo la planeación para la aplicación de acuerdo al horario de la carga académica, previamente se solicitó un tiempo de 30 minutos a los docentes que tenían asignaturas con dos horas en los grupos, para no afectar su clase. El cuestionario se alojó en Google Docs y la aplicación fue por medio de los dispositivos móviles de los alumnos; se compartió el hipervínculo del cuestionario por medio de los grupos de WhatsApp administrados por cada uno de los representantes de grupo.

El cuestionario consta de 13 ítems, los primeros diez se encuentran agrupados en las siguientes categorías de acuerdo a las Competencias Digitales Docentes (INTEF, 2017):

- Información y alfabetización informacional. Esta categoría se enfocó en identificar el nivel que ellos consideran tener al momento de seleccionar y adquirir recursos TIC, el conocimiento de componentes básicos sobre hardware y software, así como todo lo referente a conceptos que se hacen necesarios al hacer uso de las TIC y navegación en internet como, ancho de banda, conexión, velocidad, etc.

- Comunicación y colaboración. La categoría está compuesta por las respuestas dadas con relación a la frecuencia de uso de las redes sociales más utilizadas en los entornos virtuales para comunicarse, compartir recursos digitales y participación en comunidades o redes en dichos entornos.

- Creación de contenido digital. Haciendo uso de los conceptos como licencia, contenidos abiertos y derechos de autor, se tomaron en cuenta las respuestas a cinco preguntas que refieren el compartir recursos TIC, herramientas de software libre y de código abierto, la utilización de material didáctico proveniente de internet o de la misma Universidad.

- Seguridad, se obtuvo mediante las respuestas obtenidas a la frecuencia de uso en sistemas de protección tanto técnica, como de datos; así como garantizar la seguridad y privacidad de los equipos tecnológicos conociendo formas de prevención.

- Resolución de problemas. respuestas sobre preguntas hechas vinculadas al conocimiento que los alumnos consideran tener sobre las TIC con respecto a las buenas prácticas que favorecen y las posibilidades que ofrecen al enriquecimiento de estas

Los tres últimos ítems, son preguntas abiertas que indagan sobre los problemas a los que se enfrentan los estudiantes al momento de hacer uso de las TIC, la opinión que tienen sobre la formación en CD que están recibiendo por parte de la institución y un breve comentario de cómo consideran los estudiantes haber obtenido sus conocimientos y competencias relativas al uso de las TIC en el aula. 
Al aplicar el cuestionario en el aula, se seleccionaron de forma aleatoria dos de los estudiantes en cada aula y se les hizo la petición formal para que realizaran la T-autoetnografía para la investigación, se solicitó el email al cual les llegarían las instrucciones para realizarla, en un segundo momento se les envió el correo invitando a los estudiantes a reflexionar sobre las CD que ellos consideran de importancia para ser utilizadas en los ambientes de aprendizaje, los estudiantes exploraron y reflexionaron sobre las siguientes preguntas sugeridas: ¿Cómo he aprendido a utilizar la tecnología a lo largo de mi vida? ¿Cómo estoy aprendiendo ahora a utilizar la tecnología? ¿Cuándo y cómo creo que aprendo a utilizarla mejor? ¿Qué cambios señalaría como más significativos en lo que se refiere a las TIC?, las peguntas las respondieron desde su propia experiencia de vida y se podían basar en evidencias de diferentes medios y formas.

\section{RESULTADOS}

El presente trabajo aborda las CD en futuros docentes, en este entendido, era necesario indagar sobre el interés de los futuros psicopedagogos en ejercer la docencia al finalizar sus estudios para que tuviera una justificación el concepto, obteniendo como resultado que el 54.3 $\%$ respondió que sí tiene interés, el $41.1 \%$ menciona que tal vez, y solamente el $4.6 \%$ de ellos responde que no, es decir, la mayoría de los estudiantes (159 estudiantes de 175) en la Licenciatura de Psicopedagogía (LPP) al finalizar sus estudios tiene interés en ingresar al Servicio Profesional Docente en México.

Los resultados obtenidos sobre la autopercepción de la CD que aborda el cuestionario en los 10 (diez) primeros ítems agrupados en las categorías antes descritas, demuestran lo siguientes: 1) Conocimiento de Información, alfabetización informacional y técnica, en su mayoría con un nivel superficial. 2) Comunicación y colaboración, las redes sociales más usadas son WhatsApp, Facebook y YouTube. 3) Creación de contenido, dio como resultado que los alumnos la mayor fuente de material didáctico que ellos usan es proveniente de internet. 4) Seguridad, las respuestas de los alumnos muestran que algunas veces saben resolver un problema técnico y la mayoría utiliza métodos de protección como contraseñas y usuarios para garantizar la protección y privacidad de su equipo. 5) Resolución de problemas, la mayoría considera tener un conocimiento superficial (Camacho, 2020).

\subsection{FID y desarrollo de CD en la Institución}

A continuación se presenta el análisis y el resultado obtenido para las tres últimas preguntas del cuestionario aplicado. Al inicio de este bloque se hizo la pregunta sobre la formación que reciben en la institución sobre las CD para su futura práctica docente. Los resultados arrojan que la mayoría de los alumnos consideran que no se les está formando en el uso de las TIC desde su institución formadora, la respuesta obtenida como no, es de 107 (61\%) alumnos de 175 encuestados.

Se pudieron identificar algunos factores que impiden en la FID el desarrollo de las CD por medio de la respuesta de los estudiantes a la pregunta abierta: ¿Por qué consideras que en tu Universidad no te están formando correctamente para el uso de las TIC en tu futuro como docente? Las respuestas obtenidas se reunieron y analizaron por medio del software ATLAS.ti, las respuestas de los participantes se organizaron por medio de códigos agrupados por frecuencias obtenidas para diferenciar los factores de impacto en la FID en el desarrollo de las $C D$, se hizo una red con la codificación y se seleccionó diseño curricular en el software para que nos mostrara las relaciones entre cada uno de los códigos como se muestra (Figura 2). 


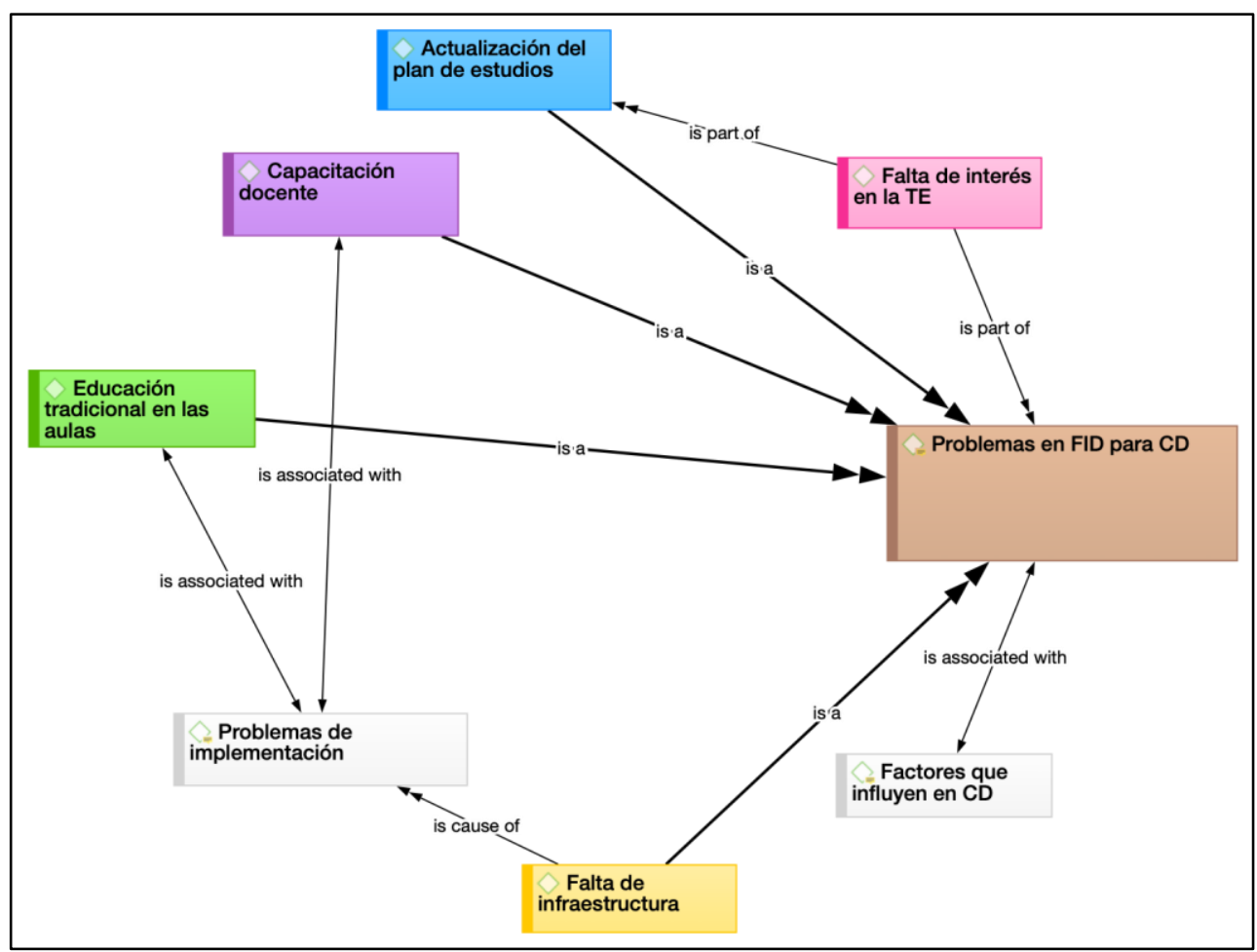

Figura 2. Red de codificación con los factores de impacto en el desarrollo de competencias digitales en la Universidad

Con el análisis antes descrito y con el proceso de codificación realizado con las respuestas de los alumnos, se obtuvo una categorización con base a la frecuencia de respuestas obtenidas de acuerdo a los factores que los alumnos identificaban y que son un factor que impide el desarrollo de las $\mathrm{CD}$, a saber:

- Educación tradicional en las aulas.

- Actualización del plan de estudios.

- Capacitación de los docentes.

- Falta de interés en la TE.

- Falta de infraestructura.

En el discurso empleado en las T-autoetnografías por parte de los estudiantes de Psicopedagogía se identifican variables que, al momento de su análisis, se pueden agrupar en Formación Inicial, Aprendizaje y TE e Impacto familiar, lo anterior se codificó por las frecuencias de las palabras mencionadas y se utilizan colores para facilitar su identificación en la agrupación (Figura 3). 


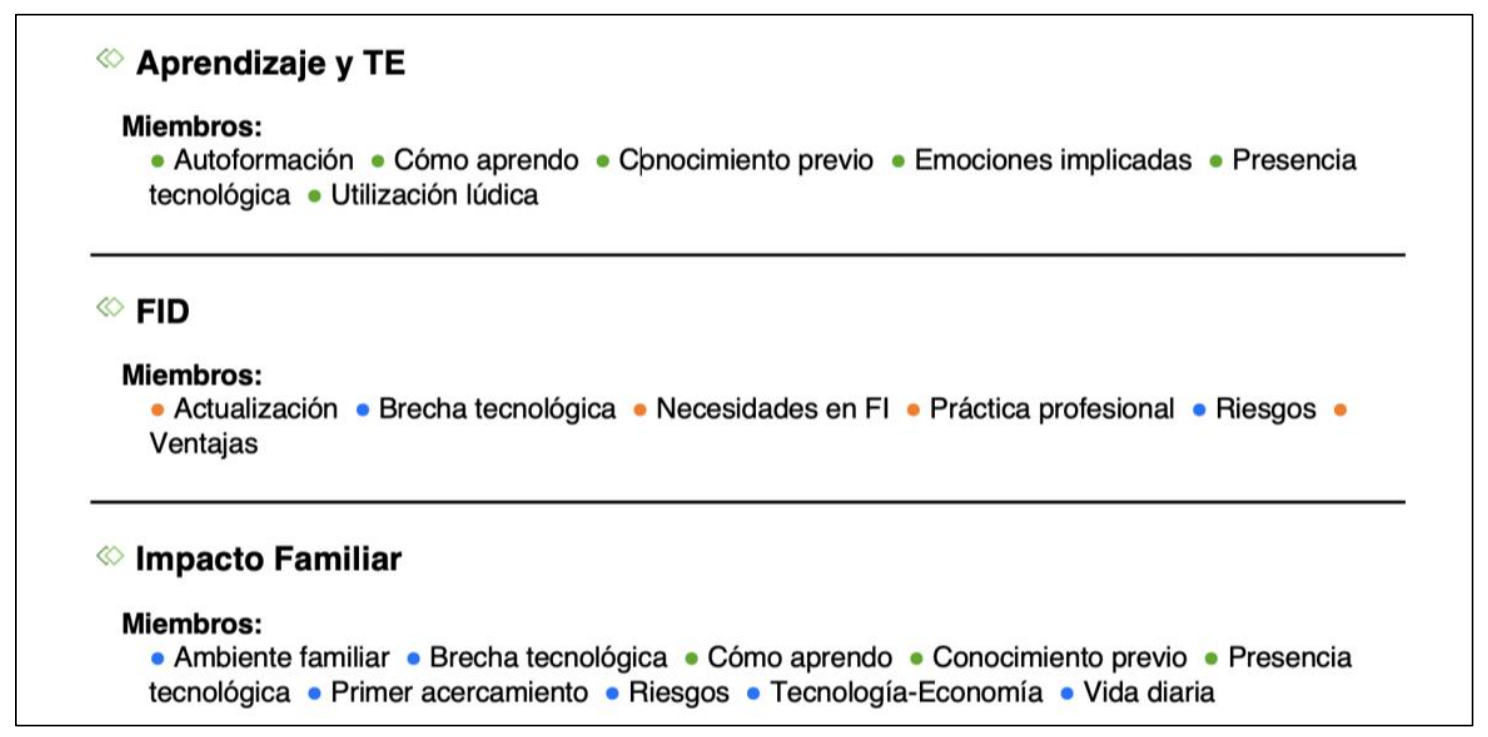

Figura 3. Lista de variables y sus códigos miembros

La organización de las variables y sus códigos pertenecientes a ellas se hicieron de acuerdo a la frecuencia de las respuestas obtenidas en las T-autoenografías y las menciones que los estudiantes hacen en ellas, las frecuencias obtenidas se agruparon por los valores abolutos de las menciones en las citas encontradas en las narrativas por semestre, algunos de estos códigos presentan un dialogo más profundo entre ellos y se presentan con más frecuencia entre los estudiantes como lo muestra (Tabla 4).

Tabla 4. Dimensiones para cada una de las categorías identificadas en las T-autoetnografías

\begin{tabular}{|c|c|c|c|c|c|}
\hline di & $\begin{array}{c}\text { Decimo } \\
\text { Semestre } \\
\text { Gr=26; } \\
\text { GS }=2^{2}\end{array}$ & $\begin{array}{c}\text { Octavo } \\
\text { Semestre } \\
\text { Gr=42; } \\
\text { GS=4 }\end{array}$ & $\begin{array}{c}\text { Segundo } \\
\text { Semestre } \\
\text { Gr=7; } \\
\text { GS=1 }\end{array}$ & $\begin{array}{c}\text { Sexto } \\
\text { Semestre } \\
\text { Gr=25; } \\
\text { GS=3 }\end{array}$ & Totales \\
\hline
\end{tabular}

$\begin{array}{cccccc}\begin{array}{c}\text { Actualización } \\ \text { Gr=2 }\end{array} & 0 & 0 & 0 & 2 & \mathbf{2} \\ \begin{array}{c}\text { ambiente familiar } \\ \text { Gr=5 }\end{array} & 1 & 2 & 1 & 1 & \mathbf{5} \\ \begin{array}{c}\text { Autoformación } \\ \text { Gr=7 }\end{array} & 1 & 3 & 0 & 3 & \mathbf{7} \\ \begin{array}{c}\text { Brecha tecnológica } \\ \text { Gr=10 }\end{array} & 5 & 3 & 0 & 2 & \mathbf{1 0} \\ \begin{array}{c}\text { Cómo aprendo } \\ \text { Gr=5 }\end{array} & 0 & 2 & 1 & 2 & \mathbf{5} \\ \begin{array}{c}\text { conocimiento previo } \\ \text { Gr=1 } \\ \begin{array}{c}\text { Emociones } \\ \text { implicadas } \\ \text { Gr=8 }\end{array}\end{array} & 0 & 1 & 0 & 0 & \mathbf{1} \\ \begin{array}{c}\text { - Necesidades en FI } \\ \text { Gr=11 }\end{array} & 3 & 5 & 0 & 2 & \mathbf{8} \\ \text { - Práctica profesional } \\ \text { Gr=4 }\end{array}$




\begin{tabular}{|c|c|c|c|c|c|}
\hline $\begin{array}{c}\text { Presencia } \\
\text { tecnológica } \\
\text { Gr=2 }\end{array}$ & 1 & 0 & 1 & 0 & 2 \\
\hline $\begin{array}{c}\text { - Primer acercamiento } \\
\text { Gr }=10\end{array}$ & 3 & 4 & 1 & 2 & 10 \\
\hline $\begin{array}{c}\text { - Riesgos } \\
\mathrm{Gr}=6\end{array}$ & 0 & 5 & 0 & 1 & 6 \\
\hline $\begin{array}{l}\text { Tecnología- } \\
\text { Economía } \\
\text { Gr=6 }\end{array}$ & 3 & 1 & 0 & 2 & 6 \\
\hline $\begin{array}{l}\text { - Utilización lúdica } \\
\qquad \mathrm{Gr}=5\end{array}$ & 1 & 2 & 1 & 1 & 5 \\
\hline $\begin{array}{l}\text { Ventajas } \\
\mathrm{Gr}=11\end{array}$ & 2 & 5 & 2 & 2 & 11 \\
\hline $\begin{array}{c}\text { - Vida diaria } \\
\text { Gr=7 }\end{array}$ & 3 & 1 & 0 & 3 & 7 \\
\hline Totales & 26 & 42 & 7 & 25 & 100 \\
\hline
\end{tabular}

Los resultados obtenidos a través del uso de ATLAS.ti arrojó la siguientes red con las tres variables (FID, Aprendizaje y TE e Impacto familiar) y los factores de impacto (códigos) que se encuentran asociados a cada una de ellas, además los resultados obtenidos también demuestran que estos códigos co-ocurren entre ellas y de las cuales se desprenden dimensiones de impacto en las expresiones de los relatos autobiográficos recopilados de forma digital, dando una opción para su estudio por las interacciones que han tenido con la TE a lo largo de su vida y que reflejan un cambio significativo para la forma de utilizar, conocer y aprender con estas herramientas (Figura 4).

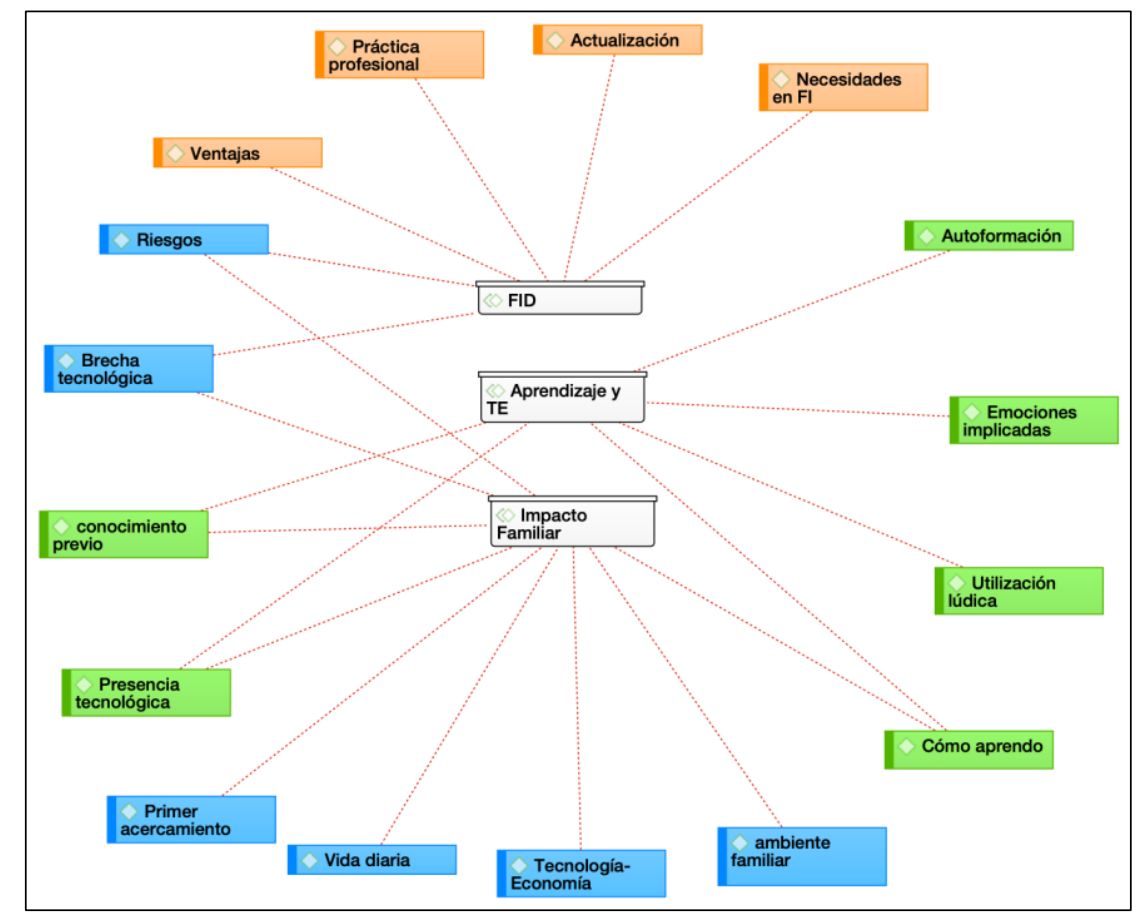

Figura 4. Red entre las variables y los factores de impacto para el desarrollo de $C D$ en los estudiantes 


\section{DISCUSIÓN Y CONCLUSIONES}

Los resultados muestran un contexto en donde se establece que la Universidad aún se trabaja de manera tradicional en la práctica docente, utilizando los mismo métodos de décadas atrás al transmitir la información en el aula; los alumnos perciben la falta de actualización docente y a su vez reclaman el uso de nuevas metodologías que incluyan la TE, de acuerdo con Escofet, Costa, \& Marimon-martí (2019), los cambios del espacio de aprendizaje se han vinculado solamente a integrar la tecnología sin hacerlo integral, sin un cambio pedagógico y ambiental.

Los futuros docentes se van a enfrentar a alumnos que conocen y manejan la tecnología de forma natural, esto no es ajeno a los estudiantes que se encuentran en FID, están preocupados y a su vez piden ser escuchados. Del mismo modo que lo señala Area Moreira (2018), mencionando existe un reclamo en el contexto o coyuntura sociocultural de incorporación de la tecnología digital a la enseñanza universitaria, así como cambios metodológicos y organizativos en la gestión de tiempo, espacios, práctica docente, contenidos, actividades de aprendizaje, evaluación y comunicación.

En el contexto mexicano todavía hay un gran rezago en el tema tecnológico, especialmente en la formación de formadores, se sigue pesando que el dotar a los futuros docentes con conocimientos pedagógicos en sus planes de estudio sin conexión con la TE es suficiente para cumplir con su futura práctica docente.

Las IES tienen un compromiso con sus estudiantes para crear espacios de aprendizaje de acuerdo al tiempo y al espacio que se vive, de tal forma que debe ir evolucionando y transformándose ligada al contexto, parte de esto es la actualización de sus planes de estudio, la capacitación docente y la adaptación de la infraestructura, sabemos que es complicado llevar a cabo estas tareas, pero se necesita hacer una planeación a corto, mediano y largo plazo.

Un gran reto es definir una metodología para el desarrollo de las $C D$ en los futuros docentes, dando voz a sus inquietudes y partiendo de los factores que ellos consideran relevantes para su práctica profesional, identificando las herramientas y metodologías que serán un apoyo para el logro de los aprendizajes significativos en sus futuros alumnos, logrando así motivarlos por medio del uso creativo e innovador de la TE en el aula.

Los hallazgos demuestran que cada agrupación realizada por la categorización necesita un nivel de análisis profundo y exhaustivo, con base a esas agrupaciones se explica lo siguiente:

a) FID, los estudiantes consideran que el estar actualizados tecnológicamente les facilitará la vida en el ámbito escolar, laboral y social y que sea parte de la FI porque la misma carrera lo demanda, expresan reiteradamente que el primer año es donde más dificultades presentan con las tareas, proyectos, presentaciones, búsqueda de información con fuentes confiables hasta la realización de su tesis y planear clases para prácticas profesionales. Consideran que la FID será la base para que en su práctica profesional puedan crear conciencia con los padres sobre el uso de la tecnología en edades tempranas y con los jóvenes sobre el uso responsable de ella. Mencionan los riesgos que ellos consideran al hacer uso de la tecnología por no estar informados. También se encuentran expresiones de los estudiantes sobre lo que ellos consideran como ventajas, el proporcionar herramientas y estrategias con las que se pueda llegar a todos los alumnos por ser útiles para todo tipo de aprendizajes a pesar de su canal de percepción de la información y la flexibilidad que representan los cursos en línea y las plataformas educativas.

b) Aprendizaje y TE, la mayoría de los alumnos han tenido que aprender de forma autónoma a utilizar la TE, mencionan que el aprendizaje también es por ayuda docente en la Universidad, seguido de esto es entre pares. Expresan la necesidad del conocimiento previo para poder realizar sus trabajos de mejor manera. En los relatos también se pueden distinguir emociones implicadas al momento de usar la TE, en su mayoría sienten miedo y que en ocasiones los maestros que impartían clases de tecnología les decían que "las iban a descomponer". Los jóvenes reconocen que la tecnología se encuentra presente en el día a día de su formación profesional. 
c) Impacto familiar, reiteradamente aparece la influencia de sus padres en el uso y acercamiento de la tecnología, ellos lo ven como su primer contacto y también como un elemento importante en su crianza. En cuanto a la brecha tecnológica ellos relacionan el nivel socioeconómico de su ambiente familiar con el acercamiento de la tecnología en sus vidas, en sus formación básica varios expresan que no había suficientes equipos de cómputo o que no tuvieron maestro, los que provienen de una comunidad agrícola estuvieron alejados de la tecnología y no tenían acceso a internet en donde era un lujo, tienen la percepción de que la educación privada incorpora mejor la TE. Consideran que pueden realizar actividades de la vida diaria gracias a la tecnología y que se pueden sustituir elementos por medios digitales.

Una T-autoetnografía presenta una riqueza en información en donde cada relato puede ser analizado desde diferentes perspectivas, se identifican elementos muy importantes que invitan a un análisis más profundo haciendo un engrane con combinaciones entre las diferentes dimensiones que al momento de interpretar la información se presentan. Al ser un relato digital y sobre una temática propuesta "la riqueza no está en la espectacularidad técnica, sino a lo que se relata, lo que implica al autor compartir su experiencia” (Roig-Vila \& Rosales-Statkus, 2016, p. 91).

Es muy importante recalcar qué gracias a la información recabada, se pueden identificar las $C D$ que los estudiantes consideran necesarias para la etapa formativa en la que se encuentran, mostrando su interés en que haya una formación en ellas en los primeros años de su carrera universitaria porque es donde más han tenido complicaciones para poder cumplir con los requerimientos que demanda su formación.

Tampoco podemos perder de vista que en los relatos aparecen las TE que ellos consideran más relevantes para poder realizar la profesión docente, siendo uno de los objetivos más importantes en esta investigación, nos proporcionan una guía de lo que se tendría que ofrecer al futuro docente como herramientas para su práctica docente. Cabero Almenara menciona que es necesario "un cambio metodológico y la transformación de las preguntas de investigación, en donde el recuerdo de la información y las experiencias de los alumnos suponen el alcance de otras variables" (2016, p. 29), por tal motivo, el análisis de las respuestas recabadas se encamina a un dialogo entre la T-autoetnografía y el investigado.

\subsection{Alcance y limitaciones}

La investigación y el diagnóstico realizado con base a las respuestas que los estudiantes han brindado, nos dan una guía de cómo puede ser una FID en CD tomando en cuenta el contexto, el obtener las impresiones de viva voz por medio de las T-autoetnografías y de las expresiones dadas a las preguntas abiertas en el cuestionario aplicado nos brinda un panorama más amplio de la realidad en la licenciatura en psicopedagogía, permite analizar el plan de estudios vigente y sus áreas de oportunidad en los temas de CD, formación en TIC, infraestructura, actualización docente, impartición de clases e integración de la TE en el aula. Partiendo del análisis se presenta una estrategia de tres materias optativas para el desarrollo de las $C D$ en la etapa formativa de los futuros psicopedagogos.

La propuesta de la estrategia está diseñada para la licenciatura de una Institución Autónoma con énfasis en pedagogía considerada en el ámbito de la FID, por ser una institución con autonomía la ley orgánica de la universidad permite la oferta de asignaturas optativas de acuerdo a las necesidades observadas, con base a un estudio exhaustivo y consensuado con las autoridades tomando en cuenta a los estudiantes.

La propuesta como estrategia para el desarrollo de las CD en estudiantes en FID que emerge de este estudio, se complica al querer adaptarlo a las Escuelas Normales, por tener un currículo rígido al ser ofertado en todas las Escuelas del país, es por esa razón que depende de las autoridades federales cualquier cambio en su malla curricular.

\section{ENLACES}

Cuestionario en línea: https://reunir.unir.net/123456789/6695 


\section{REFERENCIAS BIBLIOGRÁFICAS}

Area, M. (2018). Hacia la universidad digital: ¿dónde estamos y a dónde vamos? RIED. Revista Iberoamericana de Educación a Distancia, 21(2), 25-30. https://doi.org/10.5944/ried.21.2.21801

Bolivar, A., \& Domingo, J. (2019). La investigación ( auto ) biográfica en educación (Primera ed). Barcelona, España: octaedro.

Brun, M. (2011). Las tecnologías de la información y las comunicaciones en la formación inicial docente de América Latina. Santiago, Chile: Naciones Unidas.

Cabero, J. (2016). ¿ Qué debemos aprender de las pasadas investigaciones en Tecnología Educativa? Revista Interuniversitaria de Investigación En Tecnología Educativa, (0), 2333. https://doi.org/http://dx.doi.org/10.6018/riite/2016/256741

Camacho, A. (2020). Autopercepción de la competencia digital en futuros profesionales docentes. En M. del C. Pérez-Fuentes (Ed.), Innovación docente e investigación en educación (pp. 221-233). Madrid, España: DYKINSON.

Coll, C. (2008). Aprender y enseñar con las TIC: expectativas, realidad y potencialidades. Boletín de la Institución Libre de Enseñanza, 72, 17-40. https://doi.org/10.1097/00019442200311000-00020

Constitución Política de los Estados Unidos Mexicanos. (2020). Artículo 3ro. Diario Oficial de la Federación. Ciudad de México: Camara de Diputados del H. Congreso de la Unión. Recuperado de http://www.diputados.gob.mx/LeyesBiblio/pdf/1_080520.pdf

De Benito, B., \& Salinas, J. M. (2016). La Investigación Basada en Diseño en Tecnología Educativa. Revista Interuniversitaria de Investigación en Tecnología Educativa, (June), 4459. https://doi.org/10.6018/riite2016/260631

Escofet, A., Costa, M. L., \& Marimon-martí, M. (2019). Percepción del profesorado sobre la integración de la tecnología en el espacio escolar Teachers 'Perception of Technology Integration in Learning Spaces. Revista Interuniversitaria de Investigación en Tecnología Educativa, 37-47. https://doi.org/http://dx.doi.org/10.6018/riite.360631

Gisbert, M., González, J., \& Esteve, F. (2016). Competencia digital y competencia digital docente: una panorámica sobre el estado de la cuestión. Revista Interuniversitaria de Investigación en Tecnología Educativa, (0), 74-83. https://doi.org/10.6018/riite2016/257631

Hermann, A. A. (2015). Narrativas digitales como didácticas y estrategias de aprendizaje en los procesos de asimilación y retención del conocimiento. Sophía, 1(19), 253. https://doi.org/10.1093/ckj/stt170

Hine, C. (2012). Virtual Ethnography, 1-25. Recuperado de https://www.pcst.co/archive/pdf/Hine_PCST1994.pdf

INEE. (2017). Panorama educativo de México. Indicadores del Sistema Educativo Nacional 2016 Educación básica y media superior (Primera ed). Ciudad de México. Recuperado de www.inee.edu.mx

INTEF. (2017). Marco Común de Competencia Digital Docente - Septiembre 2017.

Larraz, V., Yáñez, C., Gisbert, M., \& Espuny, C. (2014). Experiencia interdisciplinaria en la formación inicial de maestros. Journal of New Approaches in Educational Research, 3(2), 71-78. https://doi.org/10.7821/naer.3.2.67-74

León-Gómez, A., Calderón-Garrido, D., \& Gil-Fernandez, R. (2019). The use of social networks in a Virtual University. Campus Virtuales, 8(1), 99-109. www.revistacampusvirtuales.es

Lopes, A., Hernández, F., Sancho, J. M., \& Rivas, J. I. (2013). Histórias de Vida em Educação A Construção do Conhecimento a partir de Histórias de Vi (Universita). Barcelona: ESBRINA - RECERCA. Recuperado de http://hdl.handle.net/2445/47252 
Martínez, F. (2016). Sentado en el andén. Revista Interuniversitaria de Investigación en Tecnología Educativa, (0), 17-22. https://doi.org/10.6018/riite/2016/258131

Moreno, H., \& Velázquez, R. (2012). La sociedad del conocimiento : inclusión o exclusión. Revista $\begin{array}{lcc}\text { Educación, } & 36(2), & 1-24 . \\ \text { http://www.redalyc. } \text { org/comocitar.oa?id=440248570 }\end{array}$ Recuperado de

Negroponte, N. (1995). El mundo digital. Barcelona, España: Ediciones B.

Padilla-Hernández, A. L., Gámiz-Sánchez, V. M. ., \& Romero-López, M. . A. (2018). Selección de categorías para el estudio de la evolución de la competencia digital docente del profesorado en Educación Superior. Revista Interuniversitaria de Investigación en Tecnología Educativa, 55-67. https://doi.org/10.6018/riite/2018/327881

Palacios, F. J., Gómez, M. E., \& Huertas, C. A. (2018). Formación inicial del docente AICLE en España: Retos y claves. Estudios Franco-Alemanes, 10, 141-161.

Paredes, J. (2014). Memoria de la escuela y escuela para la comunidad : análisis de un caso en formación de maestros. Tendencias Pedagógicas, 24(0), 9-22.

Prendes, M. P. (2018). La Tecnología Educativa en la Pedagogía del siglo XXI: una visión en 3D. Revista Interuniversitaria de Investigación en Tecnología Educativa, (June). https://doi.org/10.6018/riite/2018/335131

Prendes, M. P., Gutiérrez, I., \& Martínez, F. (2018). Competencia digital: una necesidad del profesorado universitario en el siglo XXI. Revista de Educación a Distancia (RED), (56), 122. https://doi.org/10.1088/0022-3735/9/10/015

Roig-Vila, R., \& Rosales-Statkus, S. (2016). El Relato Digital. Análisis de sus elementos y tipología. Revista Interuniversitaria de Investigación en Tecnología Educativa, (0), 84-94. https://doi.org/10.6018/riite/2016/257951

Sancho, J. M., Bosco, A., Alonso, C., \& Sanchez, J. (2015). Formación del profesorado en Tecnología Educativa: de cómo las realidades generan los mitos. Revista Latinoamericana de Tecnología Educativa, 14(1), 17-30. https://doi.org/10.17398/1695

SEP. (2017). Modelo Educativo (Primera). Ciudad de México: Secretaría de Educación Pública.

SEP. (2018). Acompañamiento curricular para la implementación de los Planes de Estudio 2018. Ciudad de México. https://cevie-dgespe.com/documentos/PPT_PLANES_2019.pdf

Serrano, J. L., \& Prendes, M. P. (2016). Investigar en Tecnología Educativa: ¿ por dónde empiezo ? Revista Interuniversitaria de Investigación en Tecnología Educativa, (1), 6-8. https://doi.org/http://dx.doi.org/10.6018/riite/2016/282101

Solano, I. M. (2018). Hacia un reposicionamiento de la investigación cualitativa en Tecnología Educativa. Revista Interuniversitaria de Investigación en Tecnología Educativa, (5), 8-11. https://doi.org/10.6018/riite/2018/355271

Tárraga-Mínguez, R., Sanz-Cervera, P., Pastor-Cerezuela, G., \& Fernández-Andrés, M. (2017). Análisis de la autoeficacia percibida en el uso de las TIC de futuros maestros y maestras de Educación Infantil y Educación Primaria. Revista Electrónica Interuniversitaria de Formación del Profesorado, 20(3), 107-116. https://doi.org/10.6018/reifop.20.3.263901

UNESCO. (2008). Estándares TIC para la formación inicial docente: Una propuesta en el contexto chileno. Santiago, Chile. www.unesco.cl

Vaillant, D. (2013). Integración de TIC en los sistemas de formación docente inicial y continua para la Educación Básica en América Latina (Primera). Argentina: Fondo de las Naciones Unidas para la Infancia (UNICEF). http://www.unicef.org/argentina/spanish/educacion_Integracion_TIC_sistemas_formacion_ docente.pdf

Valencia-Molina, T., Serna-Collazos, A., Ochoa-Angrino, S., Caicedo-Tamayo, A. M., MontesGonzález, J. A., \& Chávez-Vescance, J. D. (2016). Competencias y estándares TIC desde la dimensión pedagógica: una perspectiva desde los niveles de apropiación de las TIC en 
la

práctica

docente, 75. http://www.unesco.org/new/fileadmin/MULTIMEDIA/FIELD/Santiago/pdf/Competenciasestandares-TIC.pdf

Zúñiga, R. P., Lozano, P. M., García, M. M., \& Hernández, E. M. (2018). La sociedad del conocimiento y la sociedad de la información como la piedra angular en la innovación tecnológica educativa. RIDE Revista Iberoamericana para la Investigación y el Desarrollo Educativo, 8(16), 18-47. https://doi.org/10.23913/ride.v8i16.371

\section{INFORMACIÓN SOBRE LOS AUTORES}

\section{Araceli Camacho Navarro}

Universidad Autónoma de Querétaro

Estudiante de doctorado en Tecnología Educativa en la Universidad Autónoma de Querétaro, México. profesora en la Facultad de Psicología de la Universidad Autónoma de San Luis Potosí en la licenciatura de Psicopedagogía. Maestra en Creatividad e Innovación Educativa por la Universidad Autónoma de Sinaloa y Licenciada en Informática por el Instituto Tecnológico de Culiacán. Miembro de la Sociedad Mexicana de Computación en la Educación (SOMECE). Líneas de investigación: competencia digital docente, entornos virtuales de enseñanza y aprendizaje, planeación y desarrollo de programas en modalidad virtual.

\section{Rolando Javier Salinas García}

Universidad Autónoma de Querétaro

Profesor- Investigador de Tiempo Completo; Coordinador de la Unidad Multidisciplinaria de Estudios sobre el Trabajo y de la Maestría y Doctorado en Estudios Multidisciplinarios sobre el Trabajo) de la Facultad de Psicología de la Universidad Autónoma de Querétaro, México. Miembro del Registro CONACYT de Evaluadores Acreditados (RCEA). Research Affiliate en el Institute for Research in Labor and Employment (IRLE), Universidad de California, Los Ángeles (UCLA). Postdoctorado en el Institute for Research in Labor and Employment (IRLE), Universidad de California, Los Ángeles. (UCLA). Doctorado en Estudios Sociales, línea de Estudios Laborales de la UAM-I (Competencia Internacional, PNPC-CONACYT). Maestría en Psicología Social en la Universidad Autónoma de Querétaro, México. Licenciatura En Psicología del Trabajo. Universidad Autónoma de Querétaro, México.

Los textos publicados en esta revista están sujetos a una licencia de Reconocimiento 4.0 España de Creative Commons. Puede copiarlos, distribuirlos, comunicarlos públicamente y hacer obras derivadas siempre que reconozca los créditos de las obras (autoría, nombre de la revista, institución editora) de la manera especificada por los autores o por la revista. La licencia completa se puede consultar en:Licencia Creative Commons Atribución-NoComercial-Compartir por igual 4.0 Internacional. 